\title{
A Generalized System of Nonlinear Variational Inequalities in Banach Spaces
}

\author{
Prapairat Junlouchai, Anchalee Kaewcharoen, and Somyot Plubtieng \\ Department of Mathematics, Faculty of Science, Naresuan University, Phitsanulok 65000, Thailand \\ Correspondence should be addressed to Somyot Plubtieng; somyotp@nu.ac.th
}

Received 13 May 2014; Accepted 17 June 2014; Published 13 July 2014

Academic Editor: Xiaolong Qin

Copyright (C) 2014 Prapairat Junlouchai et al. This is an open access article distributed under the Creative Commons Attribution License, which permits unrestricted use, distribution, and reproduction in any medium, provided the original work is properly cited.

\begin{abstract}
We introduce a new generalized system of nonlinear variational inequality problems (GSNVIP) by using the generalized projection method. Moreover, we introduce an iterative scheme for finding a solution to this problem. Moreover, some existence and strong convergence theorems are established in uniformly smooth and strictly convex Banach spaces under suitable conditions. The results presented in the paper improve and extend some recent results.
\end{abstract}

\section{Introduction}

Variational inequality theory has become a very effective and powerful tool for studying a wide range of problems arising in pure and applied sciences which include work on differential equations, general equilibrium problems in economics and mechanics, control problems, and transportation. In 2005, Verma [1] introduced a general model for two-step projection methods and applied it to the approximation solvability of a system of nonlinear variational inequality problems in a Hilbert space. Based on the convergence of projection methods, Chang et al. [2] introduced and studied the approximate solvability of a generalized system for relaxed cocoercive nonlinear variational inequalities in Hilbert spaces (see, for instance, [3-5] and the references therein). Recently, Chang et al. [6] introduced a system of generalized nonlinear variational inequalities and an iterative scheme for finding a solution to a system of generalized nonlinear variational inequality problems by using the generalized projection method. Moreover, they proved some existence and strong convergence theorems in uniformly smooth and strictly convex Banach spaces.

In this paper, we introduce a generalized system of nonlinear variational inequality problems (GSNVIP) by using the generalized projection approach to introduce an iterative scheme for finding a solution to this problem. Finally, we prove some existence and strong convergence theorems in uniformly smooth and strictly convex Banach spaces under suitable conditions.

\section{Preliminaries}

Let $E$ be a real Banach space with dual space $E^{*},\langle\cdot, \cdot\rangle$ the dual pair between $E$ and $E^{*}$, and $K$ a nonempty closed convex subset of $E$. The normalized duality mapping $J: E \rightarrow 2^{E^{*}}$ is defined by

$$
J(x)=\left\{f^{*} \in E^{*}:\left\langle x, f^{*}\right\rangle=\|x\|^{2}=\left\|f^{*}\right\|^{2}\right\}, \quad \forall x \in E .
$$

A Banach space $E$ is said to be strictly convex if $\|x+y\| / 2<1$ for all $x, y \in U=\{z \in E:\|z\|=1\}$ with $x \neq y$. $E$ is said to be uniformly convex if for each $\epsilon \in(0,2]$ there exists $\delta>0$ such that $\|x+y\| / 2<1-\delta$ for all $x, y \in U$ with $\|x-y\| \geq \epsilon$. $E$ is said to be smooth if the limit

$$
\lim _{t \rightarrow 0} \frac{\|x+t y\|-\|x\|}{t} .
$$

exists for all $x, y \in U$. $E$ is said to be uniformly smooth if the above limit exists uniformly in $x, y \in U$. 
Remark 1 (see [7]). (i) If $E$ is a uniformly smooth Banach space, then the normalized duality mapping $J$ is uniformly continuous on each bounded subset of $E$.

(ii) If $E$ is a smooth, strictly convex and reflexive Banach space, then the normalized duality mapping $J: E \rightarrow 2^{E^{*}}$ is a single valued bijective mapping.

(iii) If $E$ is a smooth, strictly convex and reflexive Banach space and $J^{*}: E^{*} \rightarrow E$ is the duality mapping in $E^{*}$, then $J^{-1}=J^{*}, J J^{*}=I_{E^{*}}$, and $J^{*} J=I_{E}$.

(iv) If $E$ is a strictly convex and reflexive Banach space, then $J^{-1}$ is hemicontinuous; that is, $J^{-1}$ is norm-weakcontinuous.

(v) $E$ is uniformly smooth if and only if $E^{*}$ is uniformly convex.

(vi) If $E$ is a uniformly smooth and strictly convex Banach space with the Kadec-Klee property (i.e., for any sequence $\left\{x_{n}\right\} \subset E$, if $x_{n} \rightarrow x \in E$ and $\left\|x_{n}\right\| \rightarrow\|x\|$, then $x_{n} \rightarrow x$ ), then both the normalized duality mappings $J: E \rightarrow E^{*}$ and $J^{*}=J^{-1}: E^{*} \rightarrow E$ are continuous.

(vii) Each uniformly convex Banach space $E$ has the Kadec-Klee property.

Assume that $E$ is a smooth, strictly convex and reflexive Banach space and $K$ is a nonempty closed convex subset of $E$; $\phi: E \times E \rightarrow \mathbb{R}^{+}:=[0, \infty)$ to denote the Lyapunov functional defined by

$$
\phi(x, y)=\|x\|^{2}-2\langle x, J y\rangle+\|y\|^{2}, \quad \forall x, y \in E .
$$

Following Alber [8], the generalized projection $\prod_{K}$ : $E \rightarrow K$ is defined by $\prod_{K} x=z$, where $z$ is the unique solution to the minimization problem

$$
\phi(z, x)=\min _{y \in K} \phi(y, x)
$$

The existence and uniqueness of the mapping $\prod_{K}$ follow from the property of the function $\phi(x, y)$ and the strict monotonicity of the mapping $J$.

Lemma 2 (see [8]). Let $E$ be a smooth, strictly convex and reflexive Banach space and $K$ a nonempty closed convex subset of $E$. Then the following conclusions hold:

(a) if $x \in E$ and $z \in K$, then

$$
z=\prod_{K} x \Longleftrightarrow\langle y-z, J z-J x\rangle \geq 0, \quad \forall y \in K
$$

(b) $\prod_{K}$ is a continuous mapping from $E$ onto $K$.

Remark 3. If $E$ is a real Hilbert space, then $J=I$ (identity mapping), $\phi(x, y)=\|x-y\|^{2}$, and $\prod_{K}$ is the metric projection $P_{K}$ from $E$ onto $K$.

Lemma 4 (see $[9,10])$. Let $E$ be a uniformly convex Banach space, $r>0$ a positive number, and $B_{r}(0):=\{x \in E$ : $\|x\| \leq r\}$ a closed ball of $E$. Then, for any given finite subset $\left\{x_{1}, x_{2}, \ldots, x_{N}\right\} \subset B_{r}(0)$ and for any given positive numbers $\lambda_{1}, \lambda_{2}, \ldots, \lambda_{N}$ with $\sum_{n=1}^{N} \lambda_{n}=1$, there exists a continuous, strictly increasing, and convex function $g:[0,2 r) \rightarrow[0, \infty)$ with $g(0)=0$ such that for any $i, j \in\{1,2, \ldots, N\}$ with $i<j$ the following holds:

$$
\left\|\sum_{n=1}^{N} \lambda_{n} x_{n}\right\|^{2} \leq \sum_{n=1}^{N} \lambda_{n}\left\|x_{n}\right\|^{2}-\lambda_{i} \lambda_{j} g\left(\left\|x_{i}-x_{j}\right\|\right) .
$$

Lemma 5 (see [11]). Let $E$ be a real reflexive, smooth, and strictly convex Banach space. Then the following inequality holds:

$$
\|f+g\|^{2} \leq\|f\|^{2}+2\left\langle g, J^{-1}(f+g)\right\rangle, \quad \forall f, g \in E^{*} .
$$

Lemma 6 (see [6]). Let $E$ be a real Banach space, $K$ a nonempty closed convex subset of $E$ with $0 \in K$, and $\prod_{K}$ : $E \rightarrow K$ the generalized projection. Then for each $x \in E$, one has $\left\|\prod_{K} x\right\| \leq\|x\|$.

\section{Main Results}

In this section, we assume that $E$ is a real Banach space with dual space $E^{*}$ and $K$ is a nonempty closed convex subset of $E$. Let $T_{1}, \ldots, T_{N}: K^{N} \rightarrow E^{*}$ be nonlinear mappings and $f: K \rightarrow E$ a mapping. The generalized system of nonlinear variational inequality problems (GSNVIP) is to find $x_{1}^{*}, \ldots, x_{N}^{*}$ such that for all $x \in K$

$$
\begin{gathered}
\left\langle f(x)-f\left(x_{1}^{*}\right), T_{1}\left(x_{2}^{*}, x_{3}^{*}, \ldots, x_{N}^{*}, x_{1}^{*}\right)\right\rangle \geq 0, \\
\left\langle f(x)-f\left(x_{2}^{*}\right), T_{2}\left(x_{3}^{*}, x_{4}^{*}, \ldots, x_{N}^{*}, x_{1}^{*}, x_{2}^{*}\right)\right\rangle \geq 0,
\end{gathered}
$$$$
\left\langle f(x)-f\left(x_{N}^{*}\right), T_{N}\left(x_{1}^{*}, x_{2}^{*}, \ldots, x_{N-1}^{*}, x_{N}^{*}\right)\right\rangle \geq 0 .
$$

If $N=3, f=I$, and $T_{1}, T_{2}, T_{3}: K^{3} \rightarrow E^{*}$ are nonlinear mappings, then the generalized system of nonlinear variational inequality problems (GSNVIP) reduces to the following problem (see [6]) to find $x_{1}^{*}, x_{2}^{*}, x_{3}^{*}$ such that, for all $x \in K$,

$$
\begin{aligned}
& \left\langle x-x_{1}^{*}, T_{1}\left(x_{2}^{*}, x_{3}^{*}, x_{1}^{*}\right)\right\rangle \geq 0, \\
& \left\langle x-x_{2}^{*}, T_{2}\left(x_{3}^{*}, x_{1}^{*}, x_{2}^{*}\right)\right\rangle \geq 0, \\
& \left\langle x-x_{3}^{*}, T_{3}\left(x_{1}^{*}, x_{2}^{*}, x_{3}^{*}\right)\right\rangle \geq 0 .
\end{aligned}
$$

If $N=2$ and $T_{1}, T_{2}: K^{2} \rightarrow E^{*}$ are nonlinear mappings and $f: K \rightarrow E$ is a mapping, then the generalized system of nonlinear variational inequality problems (GSNVIP) reduces to the following problem to find $x_{1}^{*}, x_{2}^{*}$ such that, for all $x \in$ $K$,

$$
\begin{aligned}
& \left\langle f(x)-f\left(x_{1}^{*}\right), T_{1}\left(x_{2}^{*}, x_{1}^{*}\right)\right\rangle \geq 0, \\
& \left\langle f(x)-f\left(x_{2}^{*}\right), T_{2}\left(x_{1}^{*}, x_{2}^{*}\right)\right\rangle \geq 0 .
\end{aligned}
$$


If $T, S: K^{2} \rightarrow E^{*}$ are nonlinear mappings and $g, f:$ $K \rightarrow E$ are two mappings. Define $T_{1}, T_{2}: K^{2} \rightarrow E^{*}$ by $T_{1}\left(x_{1}^{*}, x_{2}^{*}\right)=\rho_{1} T\left(x_{1}^{*}, x_{2}^{*}\right)+g\left(x_{2}^{*}\right)-g\left(x_{1}^{*}\right)$ and $T_{2}\left(x_{1}^{*}, x_{2}^{*}\right)=$ $\rho_{2} S\left(x_{1}^{*}, x_{2}^{*}\right)+g\left(x_{2}^{*}\right)-g\left(x_{1}^{*}\right)$. Then the generalized system of nonlinear variational inequality problems (GSNVIP) reduces to the following problem to find $x_{1}^{*}, x_{2}^{*} \in K$ such that, for all $x \in K$,

$$
\begin{aligned}
& \left\langle f(x)-f\left(x_{1}^{*}\right), \rho_{1} T\left(x_{2}^{*}, x_{1}^{*}\right)+g\left(x_{2}^{*}\right)-g\left(x_{1}^{*}\right)\right\rangle \geq 0, \\
& \left\langle f(x)-f\left(x_{2}^{*}\right), \rho_{2} S\left(x_{1}^{*}, x_{2}^{*}\right)+g\left(x_{2}^{*}\right)-g\left(x_{1}^{*}\right)\right\rangle \geq 0,
\end{aligned}
$$

where $\rho_{1}$ and $\rho_{2}$ are two positive constants.

Lemma 7. Let $E$ be a smooth, strictly convex, and reflexive Banach space and $K$ a nonempty closed convex subset of $E$. Let $T_{1}, \ldots, T_{N}: K^{N} \rightarrow E^{*}$ be mappings, $f: K \rightarrow K a$ bijective mapping, and $\rho_{1}, \ldots, \rho_{N}$ any positive real numbers. Then $\left(x_{1}^{*}, \ldots, x_{N}^{*}\right) \in K^{N}$ is a solution to problem (8) if and only if $\left(x_{1}^{*}, \ldots, x_{N}^{*}\right) \in K^{N}$ is a solution to the following system of operator equations:

$$
\begin{gathered}
x_{1}^{*}=f^{-1} \prod_{K} J^{-1}\left(J f\left(x_{1}^{*}\right)-\rho_{1} T_{1}\left(x_{2}^{*}, x_{3}^{*}, \ldots, x_{N}^{*}, x_{1}^{*}\right)\right), \\
x_{2}^{*}=f^{-1} \prod_{K} J^{-1}\left(J f\left(x_{2}^{*}\right)-\rho_{2} T_{2}\left(x_{3}^{*}, x_{4}^{*}, \ldots, x_{N}^{*}, x_{1}^{*}, x_{2}^{*}\right)\right), \\
\vdots \\
x_{N-1}^{*} \\
=f^{-1} \prod_{K} J^{-1}\left(J f\left(x_{N-1}^{*}\right)\right. \\
\left.\quad-\rho_{N-1} T_{N-1}\left(x_{N}^{*}, x_{1}^{*}, x_{2}^{*}, \ldots, x_{N-2}^{*}, x_{N-1}^{*}\right)\right), \\
x_{N}^{*}=f^{-1} \prod_{K} J^{-1}\left(J f\left(x_{N}^{*}\right)-\rho_{N} T_{N}\left(x_{1}^{*}, x_{2}^{*}, \ldots, x_{N}^{*}\right)\right) .
\end{gathered}
$$

Proof. By Lemma 2, we have that $\left(x_{1}^{*}, \ldots, x_{N}^{*}\right) \in K^{N}$ is a solution of problem (8),

$$
\Longleftrightarrow\left\{\begin{array}{c}
\left\langle f(x)-f\left(x_{1}^{*}\right),\right. \\
\left.\quad \rho_{1} T_{1}\left(x_{2}^{*}, x_{3}^{*}, \ldots, x_{N}^{*}, x_{1}^{*}\right)\right\rangle \geq 0, \\
\left\langle f(x)-f\left(x_{2}^{*}\right),\right. \\
\left.\rho_{2} T_{2}\left(x_{3}^{*}, x_{4}^{*}, \ldots, x_{N}^{*}, x_{1}^{*}, x_{2}^{*}\right)\right\rangle \geq 0, \\
\vdots \\
\left\langle f(x)-f\left(x_{N-1}^{*}\right),\right. \\
\left.\rho_{N-1} T_{N-1}\left(x_{N}^{*}, x_{1}^{*}, x_{2}^{*}, \ldots, x_{N-2}^{*}, x_{N-1}^{*}\right)\right\rangle \geq 0, \\
\left\langle f(x)-f\left(x_{N}^{*}\right),\right. \\
\left.\rho_{N} T_{N}\left(x_{1}^{*}, x_{2}^{*}, \ldots, x_{N}^{*}\right)\right\rangle \geq 0,
\end{array}\right.
$$

$$
\Longleftrightarrow\left\{\begin{array}{c}
\left\langle f(x)-f\left(x_{1}^{*}\right), J f\left(x_{1}^{*}\right)-J f\left(x_{1}^{*}\right)\right. \\
\left.\quad+\rho_{1} T_{1}\left(x_{2}^{*}, x_{3}^{*}, \ldots, x_{N}^{*}, x_{1}^{*}\right)\right\rangle \geq 0, \\
\left\langle f(x)-f\left(x_{2}^{*}\right), J f\left(x_{2}^{*}\right)-J f\left(x_{2}^{*}\right)\right. \\
\left.\quad+\rho_{2} T_{2}\left(x_{3}^{*}, x_{4}^{*}, \ldots, x_{N}^{*}, x_{1}^{*}, x_{2}^{*}\right)\right\rangle \geq 0, \\
\vdots \\
\left\langle f(x)-f\left(x_{N-1}^{*}\right), J f\left(x_{N-1}^{*}\right)-J f\left(x_{N-1}^{*}\right)\right. \\
\left.\quad+\rho_{N-1} T_{N-1}\left(x_{N}^{*}, x_{1}^{*}, x_{2}^{*}, \ldots, x_{N-2}^{*}, x_{N-1}^{*}\right)\right\rangle \geq 0, \\
\left\langle f(x)-f\left(x_{N}^{*}\right), J f\left(x_{N}^{*}\right)\right. \\
\left.\quad-J f\left(x_{N}^{*}\right)+\rho_{N} T_{N}\left(x_{1}^{*}, x_{2}^{*}, \ldots, x_{N}^{*}\right)\right\rangle \geq 0,
\end{array}\right.
$$$$
\Longleftrightarrow\left\{\begin{array}{c}
\left\langle f(x)-f\left(x_{1}^{*}\right), J f\left(x_{1}^{*}\right)\right. \\
-J\left(J ^ { - 1 } \left(J f\left(x_{1}^{*}\right)\right.\right. \\
\left.\left.\left.\quad-\rho_{1} T_{1}\left(x_{2}^{*}, x_{3}^{*}, \ldots, x_{N}^{*}, x_{1}^{*}\right)\right)\right)\right\rangle \geq 0, \\
\left\langle f(x)-f\left(x_{2}^{*}\right), J f\left(x_{2}^{*}\right)\right. \\
-J\left(J ^ { - 1 } \left(J f\left(x_{2}^{*}\right)\right.\right. \\
\left.\left.\left.\quad-\rho_{2} T_{2}\left(x_{3}^{*}, x_{4}^{*}, \ldots, x_{N}^{*}, x_{1}^{*}, x_{2}^{*}\right)\right)\right)\right\rangle \geq 0, \\
\vdots \\
\left\langle f(x)-f\left(x_{N-1}^{*}\right), J f\left(x_{N-1}^{*}\right)\right. \\
-J\left(J ^ { - 1 } \left(J f\left(x_{N-1}^{*}\right)-\rho_{N-1} T_{N-1}\right.\right. \\
\left.\left.\left.\quad \times\left(x_{N}^{*}, x_{1}^{*}, x_{2}^{*}, \ldots, x_{N-2}^{*}, x_{N-1}^{*}\right)\right)\right)\right\rangle \geq 0, \\
\left\langle f(x)-f\left(x_{N}^{*}\right), J f\left(x_{N}^{*}\right)\right. \\
-J\left(J ^ { - 1 } \left(J f\left(x_{N}^{*}\right)\right.\right. \\
\left.\left.\left.\quad-\rho_{N} T_{N}\left(x_{1}^{*}, x_{2}^{*}, \ldots, x_{N}^{*}\right)\right)\right)\right\rangle \geq 0,
\end{array}\right.
$$

for all $x \in K$,

$$
\Longleftrightarrow\left\{\begin{array}{l}
f\left(x_{1}^{*}\right) \\
=\prod_{K} J^{-1}\left(J f\left(x_{1}^{*}\right)-\rho_{1} T_{1}\left(x_{2}^{*}, x_{3}^{*}, \ldots, x_{N}^{*}, x_{1}^{*}\right)\right), \\
f\left(x_{2}^{*}\right) \\
=\prod_{K} J^{-1}\left(J f\left(x_{2}^{*}\right)\right. \\
\left.\quad-\rho_{2} T_{2}\left(x_{3}^{*}, x_{4}^{*}, \ldots, x_{N}^{*}, x_{1}^{*}, x_{2}^{*}\right)\right), \\
\vdots \\
\begin{array}{l}
f\left(x_{N-1}^{*}\right) \\
=\prod_{K} J^{-1}\left(J f\left(x_{N-1}^{*}\right)\right.
\end{array} \\
\quad-\rho_{N-1} T_{N-1} \\
f\left(x_{N}^{*}\right) \\
\left.=\prod_{K} J^{-1}\left(J f\left(x_{N}^{*}\right)-x_{N}^{*}, x_{2}^{*}, \ldots, x_{N-2}^{*}, x_{N-1}^{*}\right)\right),
\end{array}\right.
$$


for any $\rho_{1}>0, \ldots, \rho_{N}>0$,

$$
\Longleftrightarrow\left\{\begin{array}{cc}
x_{1}^{*} & f^{-1} \prod_{K} J^{-1}\left(J f\left(x_{1}^{*}\right)\right. \\
& \left.-\rho_{1} T_{1}\left(x_{2}^{*}, x_{3}^{*}, \ldots, x_{N}^{*}, x_{1}^{*}\right)\right) \\
x_{2}^{*} & f^{-1} \prod_{K} J^{-1}\left(J f\left(x_{2}^{*}\right)\right. \\
& \left.-\rho_{2} T_{2}\left(x_{3}^{*}, x_{4}^{*}, \ldots, x_{N}^{*}, x_{1}^{*}, x_{2}^{*}\right)\right) \\
& \vdots \\
x_{N-1}^{*} & \\
=f^{-1} \prod_{K} J^{-1}\left(J f\left(x_{N-1}^{*}\right)-\rho_{N-1} T_{N-1}\right. & \left.\left(x_{N}^{*}, x_{1}^{*}, x_{2}^{*}, \ldots, x_{N-2}^{*}, x_{N-1}^{*}\right)\right) \\
x_{N}^{*} & \left.-\rho_{N} T_{N}\left(x_{1}^{*}, x_{2}^{*}, \ldots, x_{N}^{*}\right)\right)
\end{array}\right.
$$

Algorithm 8. For any given initial points $x_{0}^{(1)}, x_{0}^{(2)}, \ldots, x_{0}^{(N)} \in$ $K$, compute the sequences $\left\{x_{n}^{(1)}\right\},\left\{x_{n}^{(2)}\right\}, \ldots,\left\{x_{n}^{(N)}\right\}$ by the iterative processes

$$
\begin{gathered}
x_{n+1}^{(N)}=f^{-1} \\
\times\left(J ^ { - 1 } \left(\left(1-\alpha_{n}^{(N)}\right) J f\left(x_{n}^{(N)}\right)+\alpha_{n}^{(N)} J\right.\right. \\
\times\left(\prod _ { K } J ^ { - 1 } \left(J f\left(x_{n}^{(N)}\right)-\rho_{N} T_{N}\right.\right. \\
\left.\left.\left.\left.\times x_{n+1}^{(N-1) \times} f^{-1} \times\left(x_{n}^{(1)}, x_{n}^{(2)}, \ldots, x_{n}^{(N)}\right)\right)\right)\right)\right), \\
\times\left(J ^ { - 1 } \left(\left(1-\alpha_{n}^{(N-1)}\right) J f\left(x_{n}^{(N-1)}\right)+\alpha_{n}^{(N-1)} J\right.\right. \\
\times\left(\prod _ { K } J ^ { - 1 } \left(J f\left(x_{n}^{(N-1)}\right)-\rho_{N-1} T_{N-1}\right.\right. \\
\times\left(x_{n+1}^{(N)}, x_{n}^{(1)}, x_{n}^{(2)}, \ldots,\right. \\
\left.\left.\left.\left.x_{n}^{(N-2)}, x_{n}^{(N-1)}\right)\right)\right)\right)
\end{gathered}
$$$$
x_{n+1}^{(2)}=f^{-1}
$$$$
\times\left(J ^ { - 1 } \left(\left(1-\alpha_{n}^{(2)}\right) J f\left(x_{n}^{(2)}\right)+\alpha_{n}^{(2)} J\right.\right.
$$$$
\times\left(\prod _ { K } J ^ { - 1 } \left(J f\left(x_{n}^{(2)}\right)-\rho_{2} T_{2}\right.\right.
$$$$
\times\left(x_{n+1}^{(3)}, x_{n+1}^{(4)}, \ldots, x_{n+1}^{(N)},\right.
$$$$
\left.\left.\left.\left.x_{n}^{(1)}, x_{n}^{(2)}\right)\right)\right)\right) \text {, }
$$$$
x_{n+1}^{(1)}=f^{-1}
$$$$
\times\left(J ^ { - 1 } \left(\left(1-\alpha_{n}^{(1)}\right) J f\left(x_{n}^{(1)}\right)+\alpha_{n}^{(1)} J\right.\right.
$$$$
\times\left(\prod _ { K } J ^ { - 1 } \left(J f\left(x_{n}^{(1)}\right)-\rho_{1} T_{1}\right.\right.
$$$$
\times\left(x_{n+1}^{(2)}, x_{n+1}^{(3)}, \ldots,\right.
$$$$
\left.\left.\left.\left.x_{n+1}^{(N)}, x_{n}^{(1)}\right)\right)\right)\right)
$$$$
n \geq 0 \text {, }
$$

where $\prod_{K}$ is the generalized projection and $\left\{\alpha_{n}^{(1)}\right\},\left\{\alpha_{n}^{(2)}\right\}, \ldots$, $\left\{\alpha_{n}^{(N)}\right\}$ are sequences in $[0,1]$.

Theorem 9. Let $E$ be a real uniformly smooth and strictly convex Banach space with Kadec-Klee property and $K a$ nonempty closed and convex subset of $E$ with $\theta \in K$. Let $f: K \rightarrow K$ be an isometry mapping, $T_{1}, \ldots, T_{N}:$ $K^{N} \rightarrow E^{*}$ continuous mappings, and $\left\{\alpha_{n}^{(1)}\right\},\left\{\alpha_{n}^{(2)}\right\}, \ldots,\left\{\alpha_{n}^{(N)}\right\}$ the sequences in $(a, b)$ with $0<a<b<1$ satisfying the following conditions:

(i) there exist a compact subset $C \subset E^{*}$ and constants $\rho_{1}>$ $0, \rho_{2}>0, \ldots, \rho_{N}>0$ such that

$$
\begin{aligned}
\left(J(K)-\rho_{N} T_{N}\left(K^{N}\right)\right) & \cup\left(J(K)-\rho_{N-1} T_{N-1}\left(K^{N}\right)\right) \\
& \cup \cdots \cup\left(J(K)-\rho_{1} T_{1}\left(K^{N}\right)\right) \subset C,
\end{aligned}
$$


where $J\left(x_{1}, x_{2}, \ldots, x_{N}\right)=J x_{N}$, for all $\left(x_{1}, x_{2}, \ldots\right.$, $\left.x_{N}\right) \in K^{N}$, and

$$
\begin{aligned}
& \left\langle T_{1}\left(x_{1}, x_{2}, \ldots, x_{N}\right)\right. \\
& \left.J^{-1}\left(J x_{N}-\rho_{1} T_{1}\left(x_{1}, x_{2}, \ldots, x_{N}\right)\right)\right\rangle \geq 0 \\
& \left\langle T_{2}\left(x_{1}, x_{2}, \ldots, x_{N}\right)\right. \\
& \left.\quad J^{-1}\left(J x_{N}-\rho_{2} T_{2}\left(x_{1}, x_{2}, \ldots, x_{N}\right)\right)\right\rangle \geq 0 \\
& \quad \vdots \\
& \left\langle T_{N}\left(x_{1}, x_{2}, \ldots, x_{N}\right)\right. \\
& \left.\quad J^{-1}\left(J x_{N}-\rho_{N} T_{N}\left(x_{1}, x_{2}, \ldots, x_{N}\right)\right)\right\rangle \geq 0
\end{aligned}
$$

for all $x_{1}, x_{2}, \ldots, x_{N} \in K$;

(ii) $\lim _{n \rightarrow \infty} \alpha_{n}^{(1)}=d_{1} \in(a, b), \lim _{n \rightarrow \infty} \alpha_{n}^{(2)}=d_{2} \in(a, b)$, $\ldots, \lim _{n \rightarrow \infty} \alpha_{n}^{(N)}=d_{N} \in(a, b)$. Let $\left\{x_{n}^{(1)}\right\},\left\{x_{n}^{(2)}\right\}$, $\ldots,\left\{x_{n}^{(N)}\right\}$ be the sequences defined by (16). Then the problem (8) has a solution $\left(x_{1}^{*}, x_{2}^{*}, \ldots, x_{N}^{*}\right) \in K^{N}$ and the sequences $\left\{x_{n}^{(1)}\right\},\left\{x_{n}^{(2)}\right\}, \ldots,\left\{x_{n}^{(N)}\right\}$ converge strongly to $x_{1}^{*}, x_{2}^{*}, \ldots, x_{N}^{*}$, respectively.

Proof.

Step 1. We first show that the sequences $\left\{x_{n}^{(1)}\right\},\left\{x_{n}^{(2)}\right\}, \ldots$, $\left\{x_{n}^{(N)}\right\}$ are bounded in $K$. It follows from Lemma 5 where $J$ is bijective and condition (18) that

$$
\begin{aligned}
& \left\|J f\left(x_{n}^{(N)}\right)-\rho_{N} T_{N}\left(x_{n}^{(1)}, x_{n}^{(2)}, \ldots, x_{n}^{(N)}\right)\right\|^{2} \\
& \leq\left\|J f\left(x_{n}^{(N)}\right)\right\|^{2} \\
& \quad-2 \rho_{N}\left\langle T_{N}\left(x_{n}^{(1)}, x_{n}^{(2)}, \ldots, x_{n}^{(N)}\right),\right. \\
& \left.J^{-1}\left(J f\left(x_{n}^{(N)}\right)-\rho_{N} T_{N}\left(x_{n}^{(1)}, x_{n}^{(2)}, \ldots, x_{n}^{(N)}\right)\right)\right\rangle \\
& \leq\left\|J f\left(x_{n}^{(N)}\right)\right\|^{2}=\left\|f\left(x_{n}^{(N)}\right)\right\|^{2} .
\end{aligned}
$$

Similarly, we note that

$$
\begin{aligned}
& \left\|J f\left(x_{n}^{(N-1)}\right)-\rho_{N-1} T_{N-1}\left(x_{n+1}^{(N)}, x_{n}^{(1)}, \ldots, x_{n}^{(N-2)}, x_{n}^{(N-1)}\right)\right\|^{2} \\
& \leq\left\|f\left(x_{n}^{(N-1)}\right)\right\|^{2}, \\
& \| J f\left(x_{n}^{(N-2)}\right) \\
& -\rho_{N-2} T_{N-2}\left(x_{n+1}^{(N-1)}, x_{n+1}^{(N)}, x_{n}^{(1)}, \ldots, x_{n}^{(N-3)}, x_{n}^{(N-2)}\right) \|^{2} \\
& \quad \leq\left\|f\left(x_{n}^{(N-2)}\right)\right\|^{2}, \\
& \quad\left\|J f\left(x_{n}^{(2)}\right)-\rho_{2} T_{2}\left(x_{n+1}^{(3)}, x_{n+1}^{(4)}, \ldots, x_{n+1}^{(N)}, x_{n}^{(1)}, x_{n}^{(2)}\right)\right\|^{2} \\
& \leq\left\|f\left(x_{n}^{(2)}\right)\right\|^{2}, \\
& \left\|J f\left(x_{n}^{(1)}\right)-\rho_{1} T_{1}\left(x_{n+1}^{(2)}, x_{n+1}^{(3)}, \ldots, x_{n+1}^{(N)}, x_{n}^{(1)}\right)\right\|^{2} \\
& \quad \leq\left\|f\left(x_{n}^{(1)}\right)\right\|^{2} .
\end{aligned}
$$

By Lemma 6, we obtain that

$$
\begin{aligned}
& \left\|f\left(x_{n+1}^{(N)}\right)\right\| \\
& =\| f f^{-1} \\
& \times\left(J ^ { - 1 } \left(\left(1-\alpha_{n}^{(N)}\right) J f\left(x_{n}^{(N)}\right)\right.\right. \\
& +\alpha_{n}^{(N)} J\left(\prod_{K} J^{-1}\right. \\
& \times\left(J f\left(x_{n}^{(N)}\right)\right. \\
& -\rho_{N} T_{N} \\
& \left.\left.\left.\left.\times\left(x_{n}^{(1)}, x_{n}^{(2)}, \ldots, x_{n}^{(N)}\right)\right)\right)\right)\right) \\
& =\| J^{-1}\left(\left(1-\alpha_{n}^{(N)}\right) J f\left(x_{n}^{(N)}\right)\right. \\
& +\alpha_{n}^{(N)} J\left(\prod_{K} J^{-1}\right. \\
& \times\left(J f\left(x_{n}^{(N)}\right)\right. \\
& \left.\left.\left.-\rho_{N} T_{N}\left(x_{n}^{(1)}, x_{n}^{(2)}, \ldots, x_{n}^{(N)}\right)\right)\right)\right) \|
\end{aligned}
$$




$$
\begin{aligned}
=\|\left(1-\alpha_{n}^{(N)}\right) J f\left(x_{n}^{(N)}\right) & \\
& +\alpha_{n}^{(N)} J\left(\prod_{K} J^{-1}\right. \\
& \times\left(J f\left(x_{n}^{(N)}\right)\right. \\
& \left.\left.-\rho_{N} T_{N}\left(x_{n}^{(1)}, x_{n}^{(2)}, \ldots, x_{n}^{(N)}\right)\right)\right) \| \\
\leq & \left(1-\alpha_{n}^{(N)}\right)\left\|J f\left(x_{n}^{(N)}\right)\right\| \\
& +\alpha_{n}^{(N)} \| J\left(\prod_{K} J^{-1}\right. \\
& \left.\times\left(J f\left(x_{n}^{(N)}\right)-\rho_{N} T_{N}\left(x_{n}^{(1)}, x_{n}^{(2)}, \ldots, x_{n}^{(N)}\right)\right)\right) \| \\
\leq & \left(1-\alpha_{n}^{(N)}\right)\left\|J f\left(x_{n}^{(N)}\right)\right\| \\
& +\alpha_{n}^{(N)}\left\|J J^{-1}\left(J f\left(x_{n}^{(N)}\right)-\rho_{N} T_{N}\left(x_{n}^{(1)}, x_{n}^{(2)}, \ldots, x_{n}^{(N)}\right)\right)\right\| \\
= & \left(1-\alpha_{n}^{(N)}\right)\left\|f\left(x_{n}^{(N)}\right)\right\| \\
& +\alpha_{n}^{(N)}\left\|J f\left(x_{n}^{(N)}\right)-\rho_{N} T_{N}\left(x_{n}^{(1)}, x_{n}^{(2)}, \ldots, x_{n}^{(N)}\right)\right\| \\
\leq & \left(1-\alpha_{n}^{(N)}\right)\left\|f\left(x_{n}^{(N)}\right)\right\|+\alpha_{n}^{(N)}\left\|f\left(x_{n}^{(N)}\right)\right\| \\
= & \left\|f\left(x_{n}^{(N)}\right)\right\| .
\end{aligned}
$$

Since $f$ is an isometry mapping, we have $\left\|x_{n+1}^{(N)}\right\| \leq\left\|x_{n}^{(N)}\right\|$. By the same argument method as given above, we have $\left\|x_{n+1}^{(N-1)}\right\| \leq\left\|x_{n}^{(N-1)}\right\|, \ldots,\left\|x_{n+1}^{(1)}\right\| \leq\left\|x_{n}^{(1)}\right\|$. Therefore, we note that $\lim _{n \rightarrow \infty}\left\|x_{n}^{(1)}\right\|, \ldots, \lim _{n \rightarrow \infty}\left\|x_{n}^{(N)}\right\|$ exist and hence the sequences $\left\{x_{n}^{(1)}\right\},\left\{x_{n}^{(2)}\right\}, \ldots,\left\{x_{n}^{(N)}\right\}$ are bounded in $K$.

Step 2. By Lemmas 4 and 6 , where $f$ is an isometry mapping and (19), it follows that there exists a continuous strictly increasing and convex function $g:[0,2 r) \rightarrow[0, \infty)$ with $g(0)=0$ such that

$$
\begin{aligned}
& \left\|f\left(x_{n+1}^{(N)}\right)\right\|^{2} \\
& \leq\left(1-\alpha_{n}^{(N)}\right)\left\|J f\left(x_{n}^{(N)}\right)\right\|^{2} \\
& +\alpha_{n}^{(N)} \| J \prod_{K} J^{-1}\left(J f\left(x_{n}^{(N)}\right)\right. \\
& \left.\quad-\rho_{N} T_{N}\left(x_{n}^{(1)}, x_{n}^{(2)}, \ldots, x_{n}^{(N)}\right)\right) \|^{2}
\end{aligned}
$$

$$
\begin{aligned}
& -\left(1-\alpha_{n}^{(N)}\right) \alpha_{n}^{(N)} g \\
& \times\left(\| J f\left(x_{n}^{(N)}\right)\right. \\
& +J \prod_{K} J^{-1}\left(J f\left(x_{n}^{(N)}\right)\right. \\
& \left.\left.-\rho_{N} T_{N}\left(x_{n}^{(1)}, x_{n}^{(2)}, \ldots, x_{n}^{(N)}\right)\right) \|\right) \\
& \leq\left(1-\alpha_{n}^{(N)}\right)\left\|f\left(x_{n}^{(N)}\right)\right\|^{2} \\
& +\alpha_{n}^{(N)}\left\|J f\left(x_{n}^{(N)}\right)-\rho_{N} T_{N}\left(x_{n}^{(1)}, x_{n}^{(2)}, \ldots, x_{n}^{(N)}\right)\right\|^{2} \\
& -\left(1-\alpha_{n}^{(N)}\right) \alpha_{n}^{(N)} \\
& \times g\left(\| J f\left(x_{n}^{(N)}\right)\right. \\
& +J \prod_{K} J^{-1}\left(J f\left(x_{n}^{(N)}\right)\right. \\
& \left.\left.-\rho_{N} T_{N}\left(x_{n}^{(1)}, x_{n}^{(2)}, \ldots, x_{n}^{(N)}\right)\right) \|\right) \\
& \leq\left(1-\alpha_{n}^{(N)}\right)\left\|f\left(x_{n}^{(N)}\right)\right\|^{2}+\alpha_{n}^{(N)}\left\|f\left(x_{n}^{(N)}\right)\right\|^{2} \\
& -\left(1-\alpha_{n}^{(N)}\right) \alpha_{n}^{(N)} \\
& \times g\left(\| J f\left(x_{n}^{(N)}\right)\right. \\
& +J \prod_{K} J^{-1}\left(J f\left(x_{n}^{(N)}\right)\right. \\
& \left.\left.-\rho_{N} T_{N}\left(x_{n}^{(1)}, x_{n}^{(2)}, \ldots, x_{n}^{(N)}\right)\right) \|\right) \\
& =\left\|f\left(x_{n}^{(N)}\right)\right\|^{2}-\left(1-\alpha_{n}^{(N)}\right) \alpha_{n}^{(N)} \\
& \times g\left(\| J f\left(x_{n}^{(N)}\right)\right. \\
& +J \prod_{K} J^{-1}\left(J f\left(x_{n}^{(N)}\right)\right. \\
& \left.\left.-\rho_{N} T_{N}\left(x_{n}^{(1)}, x_{n}^{(2)}, \ldots, x_{n}^{(N)}\right)\right) \|\right) .
\end{aligned}
$$

This implies that

$$
\begin{gathered}
\left(1-\alpha_{n}^{(N)}\right) \alpha_{n}^{(N)} g\left(\| J f\left(x_{n}^{(1)}\right)\right. \\
+J \prod_{K} J^{-1}\left(J f\left(x_{n}^{(N)}\right)\right. \\
-\rho_{N} T_{N} \\
\left.\left.\quad \times\left(x_{n}^{(1)}, x_{n}^{(2)}, \ldots, x_{n}^{(N)}\right)\right) \|\right) \\
\leq\left\|f\left(x_{n}^{(N)}\right)\right\|^{2}-\left\|f\left(x_{n+1}^{(N)}\right)\right\|^{2} .
\end{gathered}
$$


Since $\left\{\left\|x_{n}^{(k)}\right\|\right\}$ converges for all $k=1,2, \ldots, N$, it follows by letting $n \rightarrow \infty$ in (23), condition (ii), and the property of $g$ that

$$
\begin{aligned}
& \| J f\left(x_{n}^{(N)}\right) \\
& \quad-J \prod_{K} J^{-1}\left(J f\left(x_{n}^{(N)}\right)-\rho_{N} T_{N}\left(x_{n}^{(1)}, x_{n}^{(2)}, \ldots, x_{n}^{(N)}\right)\right) \| \longrightarrow 0,
\end{aligned}
$$

as $n \rightarrow \infty$. By (16) and (24), we have

$$
\begin{aligned}
& \left\|J f\left(x_{n+1}^{(N)}\right)-J f\left(x_{n}^{(N)}\right)\right\| \\
& =\alpha_{n}^{(N)} \| J f\left(x_{n}^{(N)}\right) \\
& \quad-J \prod_{K} J^{-1}\left(J f\left(x_{n}^{(N)}\right)\right. \\
& \left.\quad-\rho_{N} T_{N}\left(x_{n}^{(1)}, x_{n}^{(2)}, \ldots, x_{n}^{(N)}\right)\right) \| \rightarrow 0,
\end{aligned}
$$

as $n \rightarrow \infty$. Similarly, we can prove that

$$
\begin{aligned}
& \left\|J f\left(x_{n+1}^{(N-1)}\right)-J f\left(x_{n}^{(N-1)}\right)\right\| \\
& =\alpha_{n}^{(N-1)} \| J f\left(x_{n}^{(N-1)}\right)-J \prod_{K} J^{-1} \\
& \times\left(J f\left(x_{n}^{(N-1)}\right)\right. \\
& -\rho_{N-1} T_{N-1} \\
& \times\left(x_{n+1}^{(N)}, x_{n}^{(1)},\right. \\
& \left.\left.x_{n}^{(2)}, \ldots, x_{n}^{(N-2)}, x_{n}^{(N-1)}\right)\right) \| \longrightarrow 0, \\
& \left\|J f\left(x_{n+1}^{(2)}\right)-J f\left(x_{n}^{(2)}\right)\right\| \\
& =\alpha_{n}^{(2)} \| J f\left(x_{n}^{(2)}\right)-J \prod_{K} J^{-1} \\
& \times\left(J f\left(x_{n}^{(2)}\right)\right. \\
& -\rho_{2} T_{2} \\
& \left.\times\left(x_{n+1}^{(3)}, x_{n+1}^{(4)}, \ldots, x_{n+1}^{(N)}, x_{n}^{(1)}, x_{n}^{(2)}\right)\right) \| \longrightarrow 0,
\end{aligned}
$$

$$
\begin{aligned}
& \left\|J f\left(x_{n+1}^{(1)}\right)-J f\left(x_{n}^{(1)}\right)\right\| \\
& =\alpha_{n}^{(1)} \| J f\left(x_{n}^{(1)}\right)-J \prod_{K} J^{-1} \\
& \quad \times\left(J f\left(x_{n}^{(1)}\right)-\rho_{1} T_{1}\right. \\
& \left.\quad \times\left(x_{n+1}^{(2)}, x_{n+1}^{(3)}, \ldots, x_{n+1}^{(N)}, x_{n}^{(1)}\right)\right) \| \longrightarrow 0,
\end{aligned}
$$

as $n \rightarrow \infty$.

Step 3. Since $\left\{x_{n}^{(1)}\right\},\left\{x_{n}^{(2)}\right\}, \ldots,\left\{x_{n}^{(N)}\right\}$ are bounded and there exists a compact subset $C \subset E^{*}$ such that $(J(K)-$ $\left.\rho_{N} T_{N}\left(K^{N}\right)\right) \subset C$, there exists a subsequence $\left\{x_{n_{i}(N)}^{(N)}\right\}$ of $\left\{x_{n_{j}}^{(N)}\right\}$ such that

$$
J f\left(x_{n_{i}(N)}^{(N)}\right)-\rho_{N} T_{N}\left(x_{n_{i}(N)}^{(1)}, x_{n_{i}(N)}^{(2)}, \ldots, x_{n_{i}(N)}^{(N)}\right) \longrightarrow h_{1} \in E^{*} .
$$

Since $E$ is uniformly smooth and strictly convex, it follows by Lemma 2 (b) and Remark 1 that $\prod_{K}$ and $J^{-1}$ are continuous. Thus

$$
\begin{aligned}
& \prod_{K} J^{-1}( J f\left(x_{n_{i}(N)}^{(N)}\right)-\rho_{N} T_{N} \\
&\left.\quad \times\left(x_{n_{i}(N)}^{(1)}, x_{n_{i}(N)}^{(2)}, \ldots, x_{n_{i}(N)}^{(N)}\right)\right) \longrightarrow \prod_{K} J^{-1}\left(h_{1}\right):=f\left(x_{N}^{*}\right),
\end{aligned}
$$

$$
\begin{aligned}
& J \prod_{K} J^{-1}\left(J f\left(x_{n_{i}(N)}^{(N)}\right)\right. \\
&\left.\quad-\rho_{N} T_{N}\left(x_{n_{i}(N)}^{(1)}, x_{n_{i}(N)}^{(2)}, \ldots, x_{n_{i}(N)}^{(N)}\right)\right) \longrightarrow J f\left(x_{N}^{*}\right) .
\end{aligned}
$$

From (24) and (29), we get

$$
J f\left(x_{n_{i}(N)}^{(N)}\right) \longrightarrow J f\left(x_{N}^{*}\right) \quad\left(\text { as } n_{i^{(N)}} \longrightarrow \infty\right) .
$$

By (25) and (30), we have

$$
J f\left(x_{n_{i}(N)+1}^{(N)}\right) \longrightarrow J f\left(x_{N}^{*}\right) \quad\left(\text { as } n_{i^{(N)}} \longrightarrow \infty\right) .
$$

Since $E$ is strictly convex and reflexive, it follows by Remark 1 (iv) that $J^{-1}$ is norm-weak-continuous. Therefore, from (30) and (31), we note that

$$
f\left(x_{n_{i}(N)}^{(N)}\right) \rightarrow f\left(x_{N}^{*}\right), \quad f\left(x_{n_{i}(N)+1}^{(N)}\right) \rightarrow f\left(x_{N}^{*}\right)
$$

and

$$
\begin{array}{r}
\left\|f\left(x_{n_{i}(N)}^{(N)}\right)\right\| \longrightarrow\left\|f\left(x_{N}^{*}\right)\right\|, \\
\left\|f\left(x_{n_{i^{(N)}}^{(N)}}^{(N)}\right)\right\| \longrightarrow\left\|f\left(x_{N}^{*}\right)\right\| \\
\left(\text { as } n_{i^{(N)}} \longrightarrow \infty\right) .
\end{array}
$$


By the Kadec-Klee property, we have

$$
\begin{array}{r}
f\left(x_{n_{i}(N)}^{(N)}\right) \rightarrow f\left(x_{N}^{*}\right), \quad f\left(x_{i_{i}(N)+1}^{(N)}\right) \\
\left(\text { as } n_{i^{(N)}} \rightarrow \infty\right) .
\end{array}
$$

Since $f^{-1}$ is continuous, it implies that $\left\{x_{n_{i}(N)}^{(N)}\right\}$ is a subsequence of $\left\{x_{n_{j}}^{(N)}\right\}$ such that $x_{n_{i}(N)}^{(N)} \rightarrow x_{N}^{*} \in E$. Therefore $x_{n}^{(N)} \rightarrow x_{N}^{*}$ as $n \rightarrow \infty$. So, it follows from (16), (30), (34), and condition (ii) that

$$
\begin{aligned}
& J f\left(x_{N}^{*}\right) \\
& =\lim _{n \rightarrow \infty} J f\left(x_{n+1}^{(N)}\right) \\
& =\lim _{n \rightarrow \infty}\left\{\left(1-\alpha_{n}^{(N)}\right) J f\left(x_{n}^{(N)}\right)+\alpha_{n}^{(N)} f^{-1} J \prod_{K} J^{-1}\right. \\
& \left.\quad \times\left(J f\left(x_{n}^{(N)}\right)-\rho_{N} T_{N}\left(x_{n}^{(1)}, x_{n}^{(2)}, \ldots, x_{n}^{(N)}\right)\right)\right\} \\
& =\left(1-d_{N}\right) J f\left(x_{N}^{*}\right) \\
& \quad+d_{N} J \prod_{K} J^{-1}\left(J f\left(x_{N}^{*}\right)-\rho_{N} T_{N}\left(x_{1}^{*}, x_{2}^{*}, \ldots, x_{N}^{*}\right)\right) .
\end{aligned}
$$

Since $f$ is a bijective mapping, we obtain that

$$
x_{N}^{*}=f^{-1} \prod_{K} J^{-1}\left(J f\left(x_{N}^{*}\right)-\rho_{N} T_{N}\left(x_{1}^{*}, x_{2}^{*}, \ldots, x_{N}^{*}\right)\right) .
$$

Similarly, we can prove that for every subsequence $\left\{x_{n_{j}}^{(k)}\right\}$ of $\left\{x_{n}^{(k)}\right\}$ there exist a subsequence $\left\{x_{n_{i}(k)}^{(k)}\right\}$ of $\left\{x_{n_{j}}^{(k)}\right\}$ and $x_{k}^{*} \in E$ such that

$$
\begin{array}{r}
f\left(x_{n_{i}(k)}^{(k)}\right) \longrightarrow f\left(x_{k}^{*}\right) \quad\left(\text { as } n_{i^{(k)}} \longrightarrow \infty\right), \\
\forall k=1,2, \ldots, N-1 .
\end{array}
$$

Since $f^{-1}$ is a continuous mapping, we note that

$$
x_{n_{i}(k)}^{(k)} \longrightarrow x_{k}^{*} \quad\left(\text { as } n_{i^{(k)}} \longrightarrow \infty\right) \text {. }
$$

Hence $x_{n}^{(k)} \rightarrow x_{k}^{*} \in E$, for all $k=1,2, \ldots, N-1$. Therefore, we have

$$
\begin{gathered}
x_{N-1}^{*}=f^{-1} \prod_{K} J^{-1}\left(J f\left(x_{N-1}^{*}\right)\right. \\
\left.-\rho_{N-1} T_{N-1}\left(x_{N}^{*}, x_{1}^{*}, \ldots, x_{N-2}^{*}, x_{N-1}^{*}\right)\right) \\
\vdots \\
x_{1}^{*}=f^{-1} \prod_{K} J^{-1}\left(J f\left(x_{1}^{*}\right)\right. \\
\left.-\rho_{1} T_{1}\left(x_{2}^{*}, x_{3}^{*}, \ldots, x_{N}^{*}, x_{1}^{*}\right)\right) .
\end{gathered}
$$

By Lemma 7 , we can conclude that $\left(x_{1}^{*}, x_{2}^{*}, \ldots, x_{N}^{*}\right)$ is a solution of (8) and $x_{n}^{(1)} \rightarrow x_{1}^{*}, x_{n}^{(2)} \rightarrow x_{2}^{*}, \ldots, x_{n}^{(N)} \rightarrow$ $x_{N}^{*}$.
Setting $N=3$ and $f=I$ in Theorem 9, we immediately obtain the following result.

Corollary 10 (see [6]). Let E be a real uniformly smooth and strictly convex Banach space with Kadec-Klee property and $K$ a nonempty closed and convex subset of $E$ with $\theta \in K$. Let $T_{1}, T_{2}, T_{3}: K^{3} \rightarrow E^{*}$ be continuous mappings and $\left\{\alpha_{n}^{(1)}\right\}$, $\left\{\alpha_{n}^{(2)}\right\}$, and $\left\{\alpha_{n}^{(3)}\right\}$ the sequences in $(a, b)$ with $0<a<b<1$ satisfying the following conditions.

(i) There exist a compact subset $C \subset E^{*}$ and constants $\rho_{1}>$ $0, \rho_{2}>0$, and $\rho_{3}>0$ such that

$$
\begin{aligned}
\left(J(K)-\rho_{3} T_{3}\left(K^{3}\right)\right) & \cup\left(J(K)-\rho_{2} T_{2}\left(K^{3}\right)\right) \\
& \cup\left(J(K)-\rho_{1} T_{1}\left(K^{3}\right)\right) \subset C,
\end{aligned}
$$

where $J\left(x_{1}, x_{2}, x_{3}\right)=J x_{3}$, for all $\left(x_{1}, x_{2}, x_{3}\right) \in K^{3}$, and $\left\langle T_{1}\left(x_{1}, x_{2}, x_{3}\right), J^{-1}\left(J x_{3}-\rho_{1} T_{1}\left(x_{1}, x_{2}, x_{3}\right)\right)\right\rangle \geq 0$,

$\left\langle T_{2}\left(x_{1}, x_{2}, x_{3}\right), J^{-1}\left(J x_{3}-\rho_{2} T_{2}\left(x_{1}, x_{2}, x_{3}\right)\right)\right\rangle \geq 0$,

$\left\langle T_{3}\left(x_{1}, x_{2}, x_{3}\right), J^{-1}\left(J x_{3}-\rho_{3} T_{3}\left(x_{1}, x_{2}, x_{3}\right)\right)\right\rangle \geq 0$,

for all $x_{1}, x_{2}, x_{3} \in K$.

(ii) $\lim _{n \rightarrow \infty} \alpha_{n}^{(1)}=d_{1} \in(a, b), \lim _{n \rightarrow \infty} \alpha_{n}^{(2)}=d_{2} \in(a, b)$, and $\lim _{n \rightarrow \infty} \alpha_{n}^{(3)}=d_{3} \in(a, b)$. Let $\left\{x_{n}^{(1)}\right\},\left\{x_{n}^{(2)}\right\}$, and $\left\{x_{n}^{(3)}\right\}$ be the sequences defined by

$x_{n+1}^{(3)}$

$$
\begin{aligned}
=J^{-1}( & \left(1-\alpha_{n}^{(3)}\right) J f\left(x_{n}^{(3)}\right)+\alpha_{n}^{(3)} J \\
& \left.\times\left(\prod_{K} J^{-1}\left(J f\left(x_{n}^{(3)}\right)-\rho_{3} T_{3}\left(x_{n}^{(1)}, x_{n}^{(2)}, x_{n}^{(3)}\right)\right)\right)\right),
\end{aligned}
$$

$x_{n+1}^{(2)}$

$$
=J^{-1}\left(\left(1-\alpha_{n}^{(2)}\right) J f\left(x_{n}^{(2)}\right)+\alpha_{n}^{(2)} J\right.
$$

$$
\left.\times\left(\prod_{K} J^{-1}\left(J f\left(x_{n}^{(2)}\right)-\rho_{2} T_{2}\left(x_{n+1}^{(3)}, x_{n}^{(1)}, x_{n}^{(2)}\right)\right)\right)\right),
$$

$x_{n+1}^{(1)}$

$$
\begin{aligned}
=J^{-1}( & \left(1-\alpha_{n}^{(1)}\right) J f\left(x_{n}^{(1)}\right)+\alpha_{n}^{(1)} J \\
& \left.\times\left(\prod_{K} J^{-1}\left(J f\left(x_{n}^{(1)}\right)-\rho_{1} T_{1}\left(x_{n+1}^{(2)}, x_{n+1}^{(3)}, x_{n}^{(1)}\right)\right)\right)\right),
\end{aligned}
$$

$n \geq 0$.

Then the problem (9) has a solution $\left(x_{1}^{*}, x_{2}^{*}, x_{3}^{*}\right) \in$ $K^{3}$ and the sequences $\left\{x_{n}^{(1)}\right\},\left\{x_{n}^{(2)}\right\}$ and $\left\{x_{n}^{(3)}\right\}$ converge strongly to $x_{1}^{*}, x_{2}^{*}$, and $x_{3}^{*}$, respectively. 
Setting $E$ as a real Hilbert space in Theorem 9, we have the following result.

Corollary 11. Let $H$ be a real Hilbert space and $K$ a nonempty closed and convex subset of $H$. Let $f: K \rightarrow K$ be an isometry mapping and $T_{1}, \ldots, T_{N}: K^{N} \rightarrow H$ continuous mappings and $\left\{\alpha_{n}^{(1)}\right\},\left\{\alpha_{n}^{(2)}\right\}, \ldots,\left\{\alpha_{n}^{(N)}\right\}$ are sequences in $(a, b)$ with $0<$ $a<b<1$ satisfying the following conditions.

(i) There exist a compact subset $C \subset H$ and constants $\rho_{1}>$ $0, \rho_{2}>0, \ldots, \rho_{N}>0$ such that

$$
\begin{aligned}
& \left(I(K)-\rho_{N} T_{N}\left(K^{N}\right)\right) \\
& \cup\left(I(K)-\rho_{N-1} T_{N-1}\left(K^{N}\right)\right) \\
& \cup \cdots \cup\left(I(K)-\rho_{1} T_{1}\left(K^{N}\right)\right) \subset C,
\end{aligned}
$$

where $\left(x_{1}, x_{2}, \ldots, x_{N}\right)=x_{N}$, for all $\left(x_{1}, x_{2}, \ldots, x_{N}\right) \in$ $K^{N}$, and

$$
\begin{gathered}
\left\langle T_{1}\left(x_{1}, x_{2}, \ldots, x_{N}\right), x_{N}-\rho_{1} T_{1}\left(x_{1}, x_{2}, \ldots, x_{N}\right)\right\rangle \geq 0 \\
\left\langle T_{2}\left(x_{1}, x_{2}, \ldots, x_{N}\right), x_{N}-\rho_{2} T_{2}\left(x_{1}, x_{2}, \ldots, x_{N}\right)\right\rangle \geq 0 \\
\vdots \\
\left\langle T_{N}\left(x_{1}, x_{2}, \ldots, x_{N}\right), x_{N}-\rho_{N} T_{N}\left(x_{1}, x_{2}, \ldots, x_{N}\right)\right\rangle \geq 0
\end{gathered}
$$

for all $x_{1}, x_{2}, \ldots, x_{N} \in K$.

(ii) $\lim _{n \rightarrow \infty} \alpha_{n}^{(1)}=d_{1} \in(a, b), \lim _{n \rightarrow \infty} \alpha_{n}^{(2)}=$ $d_{2} \in(a, b), \ldots, \lim _{n \rightarrow \infty} \alpha_{n}^{(N)}=d_{N} \in(a, b)$. Let $\left\{x_{n}^{(1)}\right\},\left\{x_{n}^{(2)}\right\}, \ldots,\left\{x_{n}^{(N)}\right\}$ be the sequences defined by

$$
\begin{aligned}
& x_{n+1}^{(N)} \\
& =f^{-1}\left(\left(1-\alpha_{n}^{(N)}\right) f\left(x_{n}^{(N)}\right)+\alpha_{n}^{(N)} P_{K}\right. \\
& \left.\times\left(f\left(x_{n}^{(N)}\right)-\rho_{N} T_{N}\left(x_{n}^{(1)}, x_{n}^{(2)}, \ldots, x_{n}^{(N)}\right)\right)\right), \\
& x_{n+1}^{(N-1)} \\
& =f^{-1}\left(\left(1-\alpha_{n}^{(N-1)}\right) f\left(x_{n}^{(N-1)}\right)+\alpha_{n}^{(N-1)} P_{K}\right. \\
& \times\left(f\left(x_{n}^{(N-1)}\right)-\rho_{N-1} T_{N-1}\right. \\
& \left.\left.\times\left(x_{n+1}^{(N)}, x_{n}^{(1)}, x_{n}^{(2)}, \ldots, x_{n}^{(N-2)}, x_{n}^{(N-1)}\right)\right)\right), \\
& x_{n+1}^{(2)} \\
& =f^{-1}\left(\left(1-\alpha_{n}^{(2)}\right) f\left(x_{n}^{(2)}\right)+\alpha_{n}^{(2)} P_{K}\right. \\
& \times\left(f\left(x_{n}^{(2)}\right)-\rho_{2} T_{2}\right. \\
& \left.\left.\times\left(x_{n+1}^{(3)}, x_{n+1}^{(4)}, \ldots, x_{n+1}^{(N)}, x_{n}^{(1)}, x_{n}^{(2)}\right)\right)\right),
\end{aligned}
$$

$$
\begin{aligned}
& x_{n+1}^{(1)} \\
& \qquad f^{-1}\left(\left(1-\alpha_{n}^{(1)}\right) f\left(x_{n}^{(1)}\right)\right. \\
& +\alpha_{n}^{(1)} P_{K}\left(f\left(x_{n}^{(1)}\right)-\rho_{1} T_{1}\right. \\
& \left.\left.\times\left(x_{n+1}^{(2)}, x_{n+1}^{(3)}, \ldots, x_{n+1}^{(N)}, x_{n}^{(1)}\right)\right)\right), \\
& n \geq 0,
\end{aligned}
$$

where $P_{K}$ is a metric projection on $H$ to $K$. Then the problem (8) has a solution $\left(x_{1}^{*}, x_{2}^{*}, \ldots, x_{N}^{*}\right) \in K^{N}$ and the sequences $\left\{x_{n}^{(1)}\right\},\left\{x_{n}^{(2)}\right\}, \ldots,\left\{x_{n}^{(N)}\right\}$ converge strongly to $x_{1}^{*}, x_{2}^{*}, \ldots, x_{N}^{*}$, respectively.

\section{Conflict of Interests}

The authors declare that there is no conflict of interests regarding the publication of this paper.

\section{Acknowledgments}

The first author would like to thank the Office of the Higher Education Commission, Thailand, for support under Grant no. CHE-Ph.D-THA-SUP/191/2551, Thailand. Moreover, the authors would like to express their deep thanks to Naresuan University under Grant no. R2557B055.

\section{References}

[1] R. U. Verma, "General convergence analysis for two-step projection methods and applications to variational problems," Applied Mathematics Letters, vol. 18, no. 11, pp. 1286-1292, 2005.

[2] S. S. Chang, H. W. Joseph Lee, and C. K. Chan, "Generalized system for relaxed cocoercive variational inequalities in Hilbert spaces," Applied Mathematics Letters, vol. 20, no. 3, pp. 329-334, 2007.

[3] X. F. He, J. M. Chen, and Z. He, "Generalized projection method for a variational inequality system with different mapping in Banach spaces," Computers \& Mathematics with Applications, vol. 58, no. 7, pp. 1391-1396, 2009.

[4] X. Qin, S. Y. Cho, and S. M. Kang, "Convergence of an iterative algorithm for systems of variational inequalities and nonexpansive mappings with applications," Journal of Computational and Applied Mathematics, vol. 233, no. 2, pp. 231-240, 2009.

[5] Q. B. Zhang and R. L. Deng, "Projection algorithms for the system of generalized mixed variational inequalities in Banach spaces," Applied Mathematics and Computation, vol. 217, no. 19, pp. 7718-7724, 2011.

[6] S. S. Chang, H. W. J. Lee, C. K. Chan, and J. A. Liu, "A new method for solving a system of generalized nonlinear variational inequalities in Banach spaces," Applied Mathematics and Computation, vol. 217, no. 16, pp. 6830-6837, 2011.

[7] I. Cioranescu, Geometry of Banach Spaces, Duality Mappings and Nonlinear Problems, Kluwer Academic Publishers, Dordrecht, Germany, 1990.

[8] Y. I. Alber, "Metric and generalized projection operators in Banach spaces: properties and applications," in Theory and Applications of Nonlinear Operators of Accretive and monotone 
type, A.G. Kartosator, Ed., vol. 178, pp. 15-50, Dekker, New York, NY, USA, 1996.

[9] S. S. Zhang, "Generalized mixed equilibrium problem in Banach spaces," Applied Mathematics and Mechanics, vol. 30, no. 9, pp. 1105-1112, 2009.

[10] H. K. Xu, "Inequalities in Banach spaces with applications," Nonlinear Analysis: Theory, Methods \& Applications, vol. 16, no. 12, pp. 1127-1138, 1991.

[11] S.-S. Chang, "On Chidume's open questions and approximate solutions of multivalued strongly accretive mapping equations in Banach spaces," Journal of Mathematical Analysis and Applications, vol. 216, no. 1, pp. 94-111, 1997. 


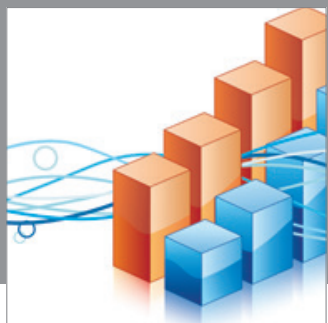

Advances in

Operations Research

mansans

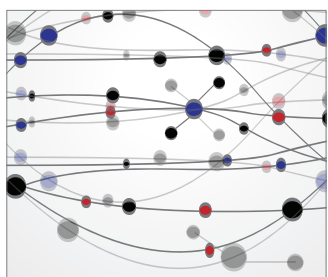

The Scientific World Journal
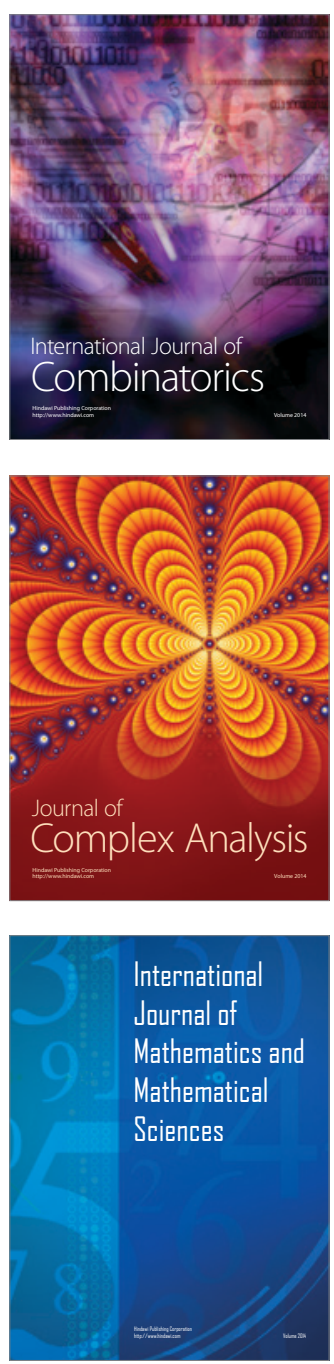
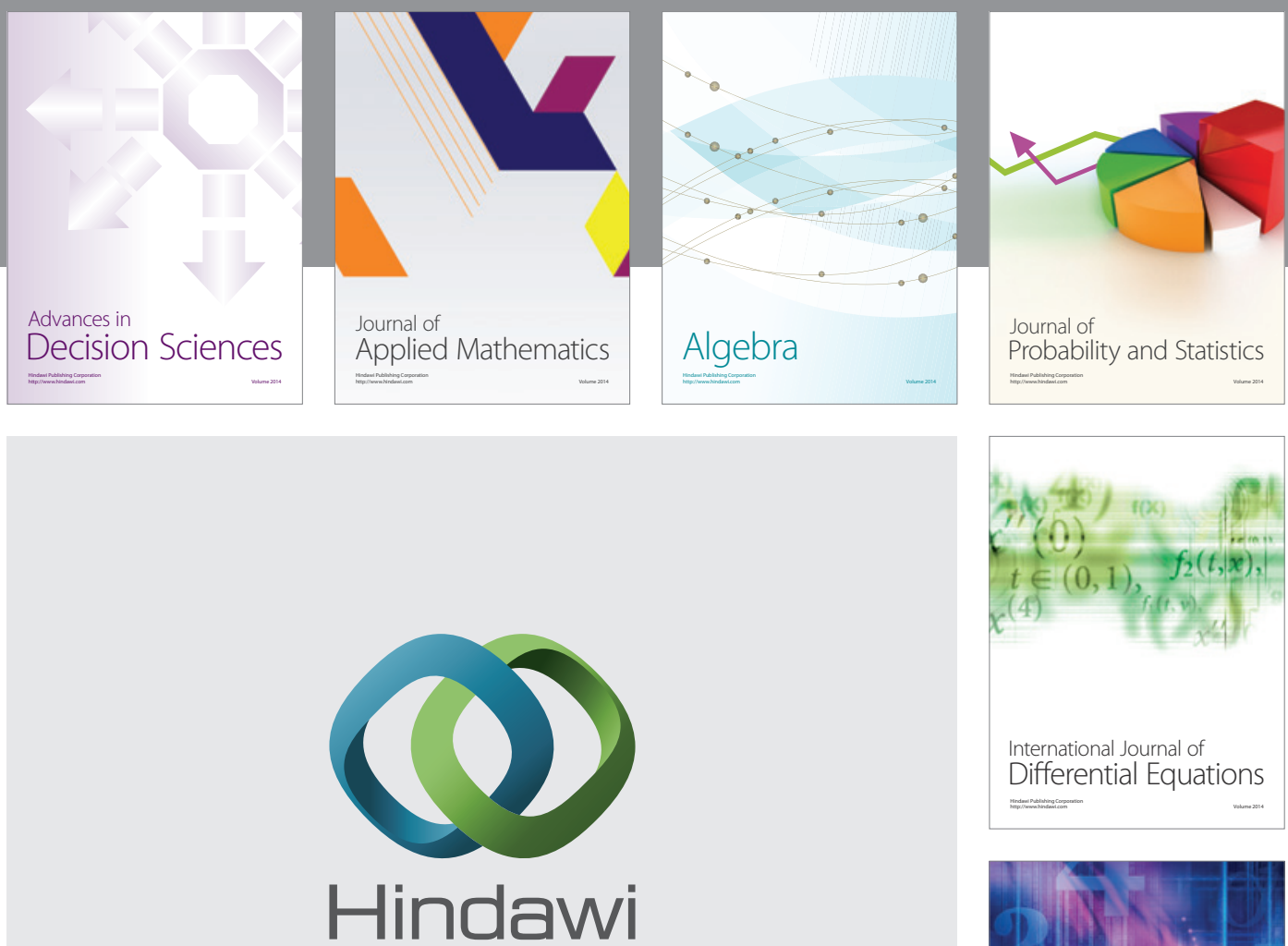

Submit your manuscripts at http://www.hindawi.com
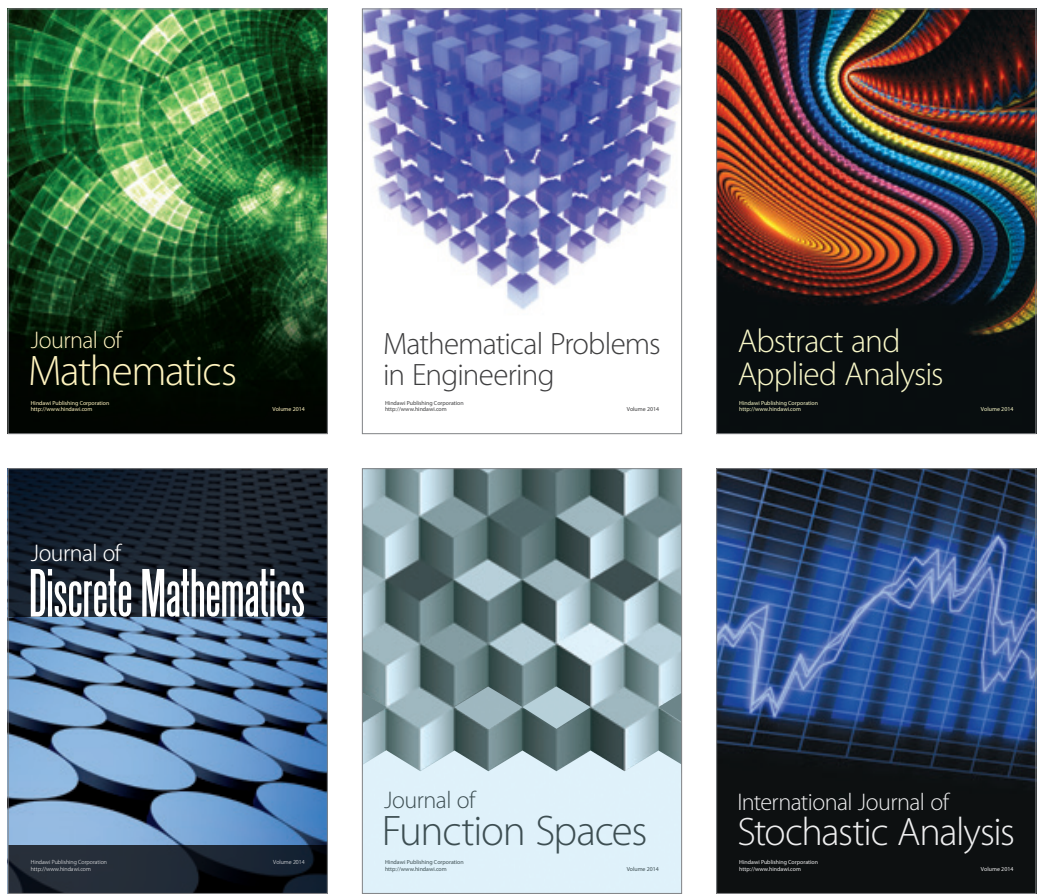

Journal of

Function Spaces

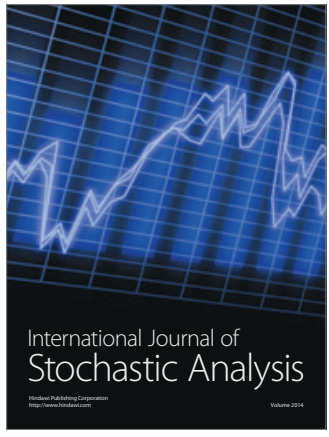

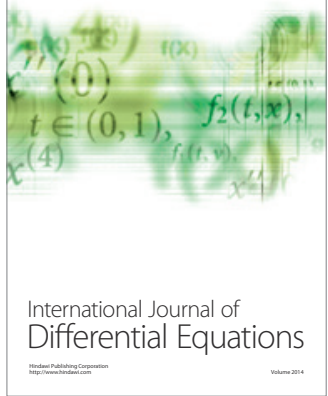
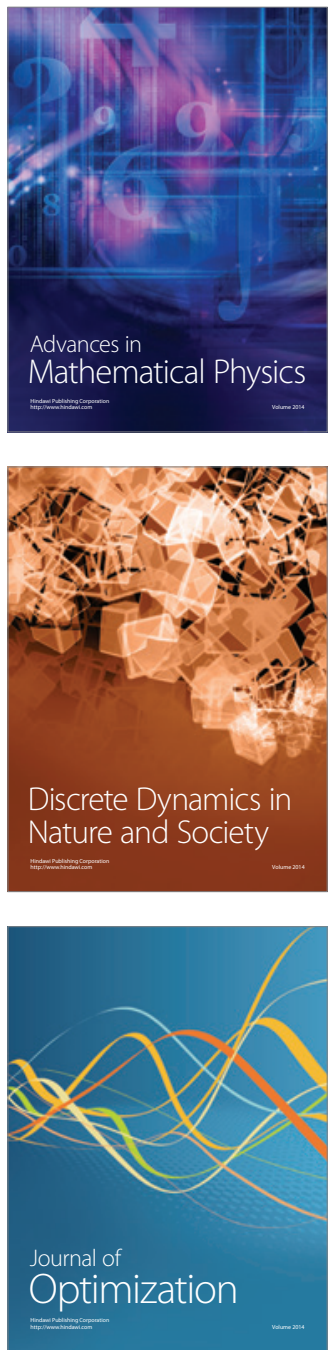